\title{
Dynamic mitral annular motion after posterior mitral annuloplasty: Use of a strip that is designed for placement in the posterior annulus
}

\author{
Ren Wen Zhang ${ }^{\mathrm{a}}$, Jong Bum Choi ${ }^{\mathrm{a}, \mathrm{b}, *}$ and Ja Hong Kuh ${ }^{\mathrm{a}, \mathrm{b}}$ \\ ${ }^{a}$ Department of Thoracic and Cardiovascular Surgery, Chonbuk National University Hospital, Jeonju, \\ Korea \\ ${ }^{\mathrm{b}}$ Research Institute of Clinical Medicine of Chonbuk National University, Biomedical Research \\ Institute of Chonbuk National University Hospital, Jeonju, Korea
}

Received 5 May 2015

Accepted 3 November 2015

\begin{abstract}
.
BACKGROUND: Most annuloplasty rings and bands used for mitral valve repair restrict annular motion due to their fixation to the posterior annulus, the commissures, and the trigones.

OBJECTIVE: Annular motion was evaluated after posterior mitral annuloplasty (PMA) for correction of mitral valve regurgitation (MR) using a strip that is designed for placement in the posterior annulus only, sparing the anterior annulus and both commissural angles.

METHODS: Valve annular dynamics were analyzed in 51 patients who underwent PMA for MR. In 37 patients $(72.5 \%)$, additional associated procedures were performed: new chord placement $(n=35)$, patch valvuloplasty $(n=4)$, and posterior leaflet augmentation $(n=12)$. Patients received serial echocardiographic follow-up.

RESULTS: After PMA, the MR grade was nil or mild ( 0 and $1+)$ in 47 patients $(92.1 \%)$, moderate (2+) in 3 patients $(5.9 \%)$, and moderate to severe $(3+)$ in 1 patient $(2.0 \%)$. The maximum and minimum septo-lateral dimensions during the cardiac cycle were $21.7 \pm 4.8 \mathrm{~mm}$ and $18.1 \pm 4.1 \mathrm{~mm}(p<0.0001)$, respectively, and the change ratios were $19.8 \pm 9.3 \%$. While the septo-lateral dimensions exhibited dynamic changes, the aorto-mitral dimensions remained constant throughout the cardiac cycle.
\end{abstract}

CONCLUSIONS: PMA preserves dynamic septo-lateral motion of the mitral valve annulus during the cardiac cycle.

Keywords: Mitral valve annuloplasty, motion/annulus

${ }^{*}$ Corresponding author: Jong Bum Choi, Department of Thoracic and Cardiovascular Surgery, Chonbuk National University Medical School, 20 Geonji-Ro, Deokjin-Gu, Jeonju, Chonbuk 561-712, Korea. Tel.: +82 63250 1486; Fax: +82 63 250 1480; E-mail: jobchoi@jbnu.ac.kr.

0928-7329/16/\$35.00 (c) 2016 - IOS Press and the authors. All rights reserved

This article is published online with Open Access and distributed under the terms of the Creative Commons Attribution NonCommercial License. 


\section{Introduction}

Most remodeling annuloplasty rings fix the annular dimensions of the mitral valve (MV) in a static position [1]. The partial flexible bands also restrict the motion at the commissural hinge, due to their fixation to the posterior annulus, the commissures, and the trigones. The commonly performed annuloplasty techniques can restore competence of the valve, but they compromise the mitral annular motion. In addition, because the annulus is contained in a flat plane of the annuloplasty ring or band, it loses both the commissural hinge action and the normal three-dimensional saddle shape [2]3]. Because most of the annular dilatation in the mitral valve occurs in the posterior annulus, we performed posterior mitral annuloplasty (PMA) using a strip that has been designed to spare the anterior annulus and the anterior half of the commissures. We evaluated the clinical outcomes after PMA procedure, and postoperatively analyzed the dynamic changes in annular dimensions during the cardiac cycle using serial follow-up echocardiograms.

\section{Materials and methods}

This study is a retrospective review of the prospective follow-up of patients who underwent PMA to address mitral valve regurgitation (MR) using a characteristic annuloplasty strip for MV repair. This study was approved by the Institutional Review Board at Chonbuk National University Hospital.

\subsection{Patients}

From September 2009 to October 2013, 51 consecutive patients ( 25 men and 26 women, mean age of $59.6 \pm 15.4$ years) presenting with greater than moderate-to-severe MR underwent PMA, in which an annuloplasty strip was used (Table 1). Patients who also underwent associated aortic valve surgery or mitral commissurotomy were excluded from this study. Procedures associated with MV repair were performed in 37 patients (72.5\%) as follows: new chord placement in 18 patients (35.3\%) with anterior leaflet prolapse, 14 patients (27.5\%) with posterior leaflet prolapse and 3 patients (5.9\%) with commissural prolapse; patch valvuloplasty [4] in 4 patients (7.8\%) with posterior chord rupture; and posterior leaflet augmentation in 12 patients $(23.5 \%)$ with a tethered or short posterior leaflet. Other concomitant procedures included the Cox-maze procedure $(n=20)$, coronary artery bypass grafting $(n=8)$, and ascending aortic aneurysm repair ( $n=3$, tangential resection).

\subsection{Posterior mitral annuloplasty strip}

The PMA strip (Mitra-Lift ${ }^{\circledR}$ strip, Scien-City, Inc., Seoul, ROK) (Fig. 1A) is a flat 5.0-mm-wide Dacron strip with two thick margins and a thin central gully. The strip is flat when straight, but when the strip is curved the middle portion becomes lifted (Fig. 1B). The strip is placed along the posterior annulus, sparing the anterior annulus and the anterior half of both commissural annuli. Placement of the strip on the plane of the atrial wall along the posterior annulus results in a curvilinear complex that lifts the middle of the posterior annulus (Fig. 1B-b), which then becomes the highest portion of the posterior annulus. Because the commissural angles are not fixed, it was expected that use of the strip would preserve the commissural hinges between two leaflets. In addition, as the strip tends to be straightened by its two thick margins, the septo-lateral annular dimensions can be reduced and the commissural hinge angles decreased. The length of the strip was determined by the intercommissural distance measured in the individual patient $(30,32,34$, or $36 \mathrm{~mm})$; the length of the strips used were $53,55,58$, and $61 \mathrm{~mm}$, respectively. 
Table 1

Perioperative data for 51 patients undergoing posterior mitral annuloplasty (PMA) for mitral valve regurgitation (MR)

\begin{tabular}{|c|c|}
\hline Characteristic & Value \\
\hline Male gender (number, \%) & $25(49 \%)$ \\
\hline Age (years) & $59.6 \pm 15.4$ \\
\hline $\operatorname{BSA}\left(\mathrm{m}^{2}\right)$ & $1.64 \pm 0.20$ \\
\hline NYHA III and IV & $46(90.2 \%)$ \\
\hline Preoperative LVEF (\%) & $56.3 \pm 9.6$ \\
\hline $\mathrm{PAP}(\mathrm{mmHg})$ & $43.0 \pm 16.9$ \\
\hline \multicolumn{2}{|l|}{ Cause of MR } \\
\hline Degenerative (number, \%) & $36(70.6 \%)$ \\
\hline Rheumatic (number, \%) & $4(7.8 \%)$ \\
\hline Ischemic (number, \%) & $7(13.7 \%)$ \\
\hline Acute chord rupture (number, \%) & $3(5.9 \%)$ \\
\hline Chronic inflammation (myeloproliferative) (number, \%) & $1(2.0 \%)$ \\
\hline \multicolumn{2}{|l|}{ Preoperative MR grade } \\
\hline $3+$, moderate-to-severe (number, \%) & $17(33.3 \%)$ \\
\hline $4+$, severe (number, $\%)$ & $34(66.7 \%)$ \\
\hline Follow-up (months) & $41.3 \pm 15.3$ \\
\hline \multicolumn{2}{|l|}{ MR grade, early postoperative } \\
\hline 0 , absent or trivial (number, $\%$ ) & $44(86.3 \%)$ \\
\hline+1, mild (number, $\%$ ) & $5(9.8 \%)$ \\
\hline+2, moderate (number, $\%$ ) & $1(2.0 \%)$ \\
\hline+3 , moderate to severe (number, $\%$ ) & $1(2.0 \%)$ \\
\hline \multicolumn{2}{|l|}{ MR grade, the latest } \\
\hline 0 , absent or trivial (number, \%) & $43(84.3 \%)$ \\
\hline+1 , mild (number, $\%$ ) & $5(9.8 \%)$ \\
\hline+2 , moderate (number, $\%$ ) & $3(5.9 \%)$ \\
\hline $\operatorname{MVA}\left(\mathrm{cm}^{2}\right)$ & $2.6 \pm 0.4$ \\
\hline Index MVA $\left(\mathrm{cm}^{2} / \mathrm{m}^{2}\right)$ & $1.6 \pm 0.3$ \\
\hline Valve gradient $(\mathrm{mmHg})$ & $3.4 \pm 1.2$ \\
\hline Leaflet coaptation depth (mm) & $4.8 \pm 1.5$ \\
\hline Leaflet coaptation height (mm) & $5.9 \pm 0.8$ \\
\hline
\end{tabular}

Data are given as $\mathrm{n}(\%)$ or as mean \pm SD. BSA, body surface area; LVEF, left ventricular ejection fraction; MR, mitral valve regurgitation; MVA, mitral valve orifice area; PAP, pulmonary artery pressure.

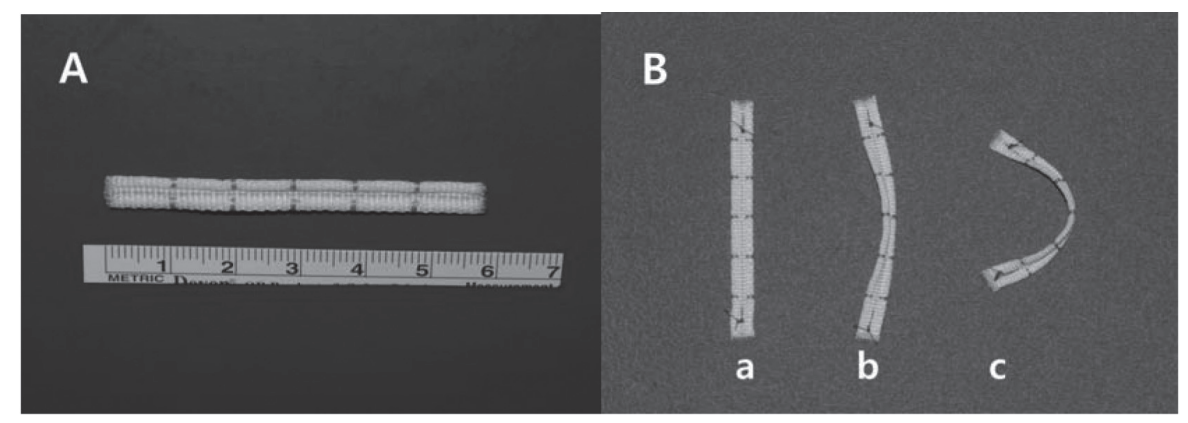

Fig. 1. (A) A posterior mitral annuloplasty strip with two thick margins and a thin middle valley. (B) The flexible strip is flat when it is straight (a), however, the middle portion is lifted by its characteristic structure when placed in a curved position in the posterior annulus $(\mathrm{b}, \mathrm{c})$.

\subsection{Surgical procedure}

The PMA procedure has previously been described in detail [5]. The MV was exposed by a left atriotomy or an interatrial septotomy under cardiopulmonary bypass with moderate hypothermia. Prior 


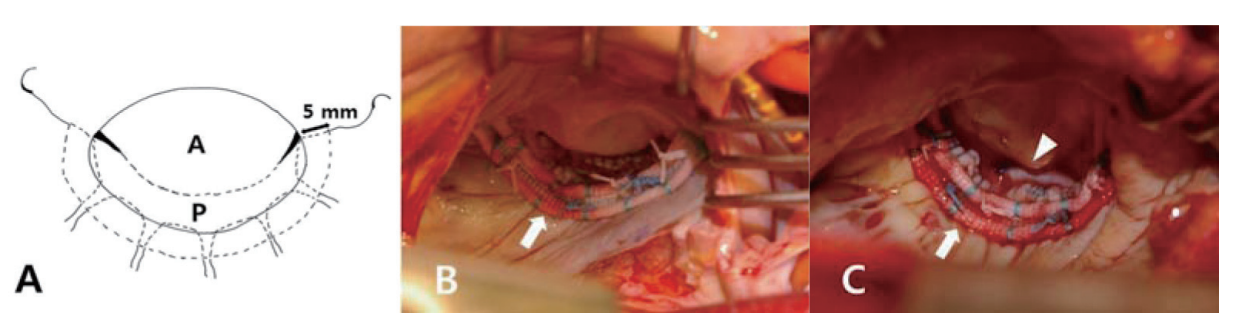

Fig. 2. (A) The strip is placed using six interrupted 2-0 Dacron mattress sutures that are passed through the 5-mm left atrial wall and the posterior annulus. On the drawing, A indicates anterior leaflet and $\mathrm{P}$ indicates posterior leaflet. (B) In cases with rheumatic mitral regurgitation, posterior leaflet augmentation and strip annuloplasty (white arrow) were performed. (C) In the saline test, the leaflet coaptation (white arrowhead) was placed below the strip (white arrow).

to PMA, new chord placement was performed in patients with anterior or posterior leaflet prolapse [6]. The length of the strip to be used was first determined by measurement of the intercommissural distance, and then six braided 2-0 Dacron sutures were passed in inverted U-shapes through the 5-mm-long supraannular atrial wall and the posterior annulus, from commissure to commissure (Fig. 2A). Both of the end sutures were placed at the middle portions of the commissural annulus. The sutures were passed through the middle gully of the strip and then tied. Upon placement of the strip on the atrial wall along the posterior annulus, the annulus and the strip become curvilinear due to the circular force applied by the annulus (Fig. 2B). In a saline test, the leaflet coaptation line was located below the strip, rather than at strip level (Fig. 2C). In cases with tethered posterior leaflets, associated with rheumatic valve disease or ischemic MR, or with a narrow posterior leaflet of $<10 \mathrm{~mm}$ in height, posterior leaflet augmentation was performed to create sufficient leaflet area for coaptation. The posterior leaflet was detached from the posterior annulus, leaving 3 to $5 \mathrm{~mm}$ of intact leaflet tissue at both ends of the posterior leaflet, and the defect was augmented with an elliptical bovine pericardial patch of $15 \mathrm{~mm} \times 45 \mathrm{~mm}$, using running 5-0 polypropylene sutures.

\subsection{Echocardiographic measurements}

Transthoracic echocardiography was performed upon admission, at discharge, at 6 months postoperatively, and annually thereafter. The MR grade was determined according to the following severity scale: 0 , no or trivial MR; $1+$, mild MR; 2+, moderate MR; 3+, moderately severe MR; and 4+, severe MR. The MV orifice area was assessed using the pressure half-time method. In the parasternal long-axis view, the preoperative and postoperative maximum and minimum septo-lateral dimensions and the aorto-mitral dimensions were measured during the cardiac cycle. The coaptation height (i.e., the longest coaptation length of the anterior and posterior leaflets) and depth (i.e., the distance between the annular plane and the uppermost coaptation point) were both measured in early systole.

\subsection{Statistical analysis}

All statistical analyses were performed using SPSS 18.0 (IBM, Armonk, NY, USA). Continuous variables were expressed as the mean \pm standard deviation and were compared using the Student's $t$-test and paired $t$-test. Categorical variables were expressed as proportions (\%) and were compared using the $\chi^{2}$ test. 
Table 2

Preoperative and postoperative annular dimensions in the parasternal long-axis view

\begin{tabular}{llll}
\hline & Preoperative dimensions & Postoperative dimensions & $P$ value \\
\hline In diastole $(\mathrm{max})(\mathrm{mm})$ & $34.1 \pm 6.7$ & $21.7 \pm 4.8$ & $<0.0001$ \\
In systole $(\mathrm{min})(\mathrm{mm})$ & $28.8 \pm 6.5$ & $18.1 \pm 4.1$ & $<0.0001$ \\
$P$ value & $<0.0001$ & $<0.0001$ & \\
$\begin{array}{l}\text { Dynamic change }(\%) \\
\text { (max-min/min) }\end{array}$ & $18.8 \pm 11.4$ & $19.8 \pm 9.3$ & 0.194 \\
Aorto-mitral dimensions $(\mathrm{mm})$ & $52.5 \pm 7.6$ & $40.9 \pm 6.0$ & $<0.0001$ \\
\hline
\end{tabular}

Data are given as $\mathrm{n}(\%)$ or mean \pm SD. Max, maximum dimension; min, minimum dimension.

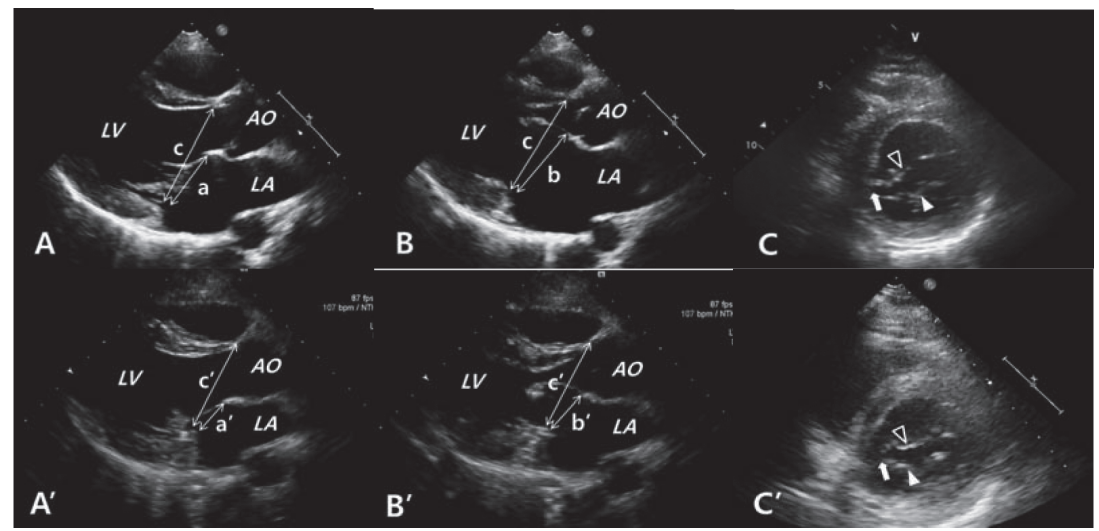

Fig. 3. Preoperative (A, B) and postoperative (A', B') echocardiograms of a patient who underwent new chord placement for posterior leaflet prolapse and posterior mitral annuloplasty. In the parasternal long-axis views, the septo-lateral dimensions were reduced in systole $\left(a \rightarrow a^{\prime}\right)$ and diastole $\left(b \rightarrow b^{\prime}\right)$ after posterior mitral annuloplasty. Preoperatively and postoperatively, dynamic changes in the septo-lateral dimensions $\left(\mathrm{a} \rightarrow \mathrm{b} ; \mathrm{a}^{\prime} \rightarrow \mathrm{b}^{\prime}\right)$ are observed during cardiac cycles, whereas the aorto-mitral dimensions (c and c') remain nearly constant during the cardiac cycle. AO, aorta; LA, left atrium; LV, left ventricle.

\section{Results}

The mean follow-up period was $41.3 \pm 15.3$ months. All patients were in New York Heart Association class I or II. The MR grade upon final measurement was determined to be absent or trivial in 43 patients $(84.3 \%)$, mild in 5 patients $(9.8 \%)$, and moderate in 3 patients $(5.9 \%)$. There was no significant progression of the MR grade observed during the follow-up period (comparison of MR grades at discharge and final follow-up; $P=0.376$; Table 2). Echocardiographic follow-up revealed MV area and MV area index of $2.6 \pm 0.4 \mathrm{~cm}^{2}$ and $1.6 \pm 0.3 \mathrm{~cm}^{2} / \mathrm{m}^{2}$, respectively, and the mean transvalvular pressure gradient was found to be $3.4 \pm 1.2 \mathrm{mmHg}$. The preoperative ratio of the maximum and minimum septo-lateral dimensions during the cardiac cycle was $18.8 \pm 11.4 \%$ (minimum of $28.8 \pm 6.5 \mathrm{~mm}$ vs. maximum of $34.1 \pm 6.7 \mathrm{~mm} ; P<0.0001$ ) (Figs 3-A and B), and the postoperative ratio of the maximum and minimum septo-lateral dimensions during the cardiac cycle was $19.8 \pm 9.3 \%$ (minimum of $18.1 \pm 9.3 \mathrm{~mm}$ vs. maximum of $21.7 \pm 4.8 \mathrm{~mm} ; P<0.0001$; Table 2; Figs 3-A' and B'). While the septo-lateral dimensions and the aorto-mitral dimensions were decreased by PMA, the dynamic ratios of the septo-lateral dimensions during the cardiac cycle were not changed after PMA (preoperative ratio vs. postoperative ratio, $18.8 \pm 11.4 \%$ vs. $19.8 \pm 9.3 \% ; P=0.194$ ) (Table 2 ). The mean leaflet coaptation depth and height observed in early systole were $4.8 \pm 1.5 \mathrm{~mm}$ and $5.9 \pm 0.8 \mathrm{~mm}$, respectively. None of patients with mild to moderate MR experienced hemolysis, and none of the patients required edge-to-edge repair or additional surgery for re-repair or for valve replacement due to remnant regurgitation. 


\section{Discussion}

The PMA procedure spares the anterior annulus and the commissural hinges, which can then maintain their usual motion during the cardiac cycle. Two mitral leaflets can achieve competent coaptation by reduction of the septo-lateral annular dimensions without reduction of the transverse annular dimensions [7]. Transverse annular reduction may actually disturb the septo-lateral coaptation of two leaflets. In our study, maintenance of the dynamic changes in the septo-lateral annular dimensions was demonstrated after PMA. Most of the rigid or partial rings that are used in annuloplasty procedures improve leaflet coaptation, but they prevent the normal annular motion. In contrast, PMA preserves the anterior annular motion and commissural hinge motion.

The middle of the strip used in PMA is lifted by two thick margins when the strip is in a curvilinear position. As a result, the middle of the posterior annulus-strip complex is lifted on the base of both commissure levels. The GeoForm annuloplasty ring (Edwards Lifesciences, Irvine, CA, USA) [8], which is used for repair of ischemic MR, also exhibited a similar coaptation-enhancing mechanism and reduction of the septo-lateral dimensions, but its rigidity also reduces the transverse dimensions and it fixes the entire annulus.

The two thick margins of the PMA strip tend to push the commissures to the outside, which narrows the commissural angle and results in stable commissural coaptation. The commissures are spared from the strip fixation, and this allows preservation of the hinge mechanism in systole. No commissural dilatation or prolapse was observed to be caused by the partially spared commissures and completely spared trigones during the follow-up period.

When the usual annuloplasty band is placed in the same fashion without involvement of both trigones and commissures, it cannot produce the narrow commissural angle and there is consequent risk of commissural dilatation or prolapse.

In patients with rheumatic or ischemic MR or narrow posterior leaflet, the posterior leaflet was augmented with an elliptical pericardial patch to obtain a sufficient coaptation area prior to PMA [9]. MR that was caused by leaflet tethering [10] could be readily repaired by the combination of posterior leaflet augmentation and the PMA procedure. In cases with posterior leaflet prolapse, we performed new chord placement [6] or patch valvuloplasty [4], rather than triangular or quadrangular resection of the prolapsed segment.

Posterior leaflet augmentation may facilitate the systolic anterior motion due to the increased posterior leaflet height. However, as the increased posterior leaflet height never affects the anterior leaflet motion after PMA, there is no risk of anterior systolic motion.

The supraannular position of the strip avoids any risk of hemolysis that is caused by remnant regurgitant flow.

There are some limitations of this study. Three-dimensional echocardiographic imaging was not performed to demonstrate the lifted posterior annulus and the preservation of commissural hinge motion. In addition, this study did not include comparison with the outcomes of procedures using the other usual rings or bands.

\section{Conclusions}

The posterior mitral annuloplasty technique uses a strip that is designed for placement in the posterior annulus only, thereby allowing the mitral annulus to maintain dynamic changes in the septo-lateral annular dimensions during the cardiac cycle. 


\section{Conflict of interest}

All of the authors declare no conflict of interest.

\section{References}

[1] Filsoufi F, Chikwe J, Adams DH. Acquired disease of the mitral valve. In: Sellke FW, del Nido PJ, Swanson SJ. Sabiston \& Spenser Surgery of the chest. 8th ed. Philadephia: Saunders Elsevier; 2010: 1221.

[2] Vergnat M, Levack MM, Jassar AS, Jackson BM, Acker MA, Woo YJ, et al. The influence of saddle-shaped annuloplasty on leaflet curvature in patients with ischaemic mitral regurgitation. Eur J Cardiothorac Surg. 2012; 42(3): 493-9.

[3] Ryomoto M, Mitsuno M, Yamamura M, Tanaka H, Fukui S, Tsujiya N, et al. Is physiologic annular dynamics preserved after mitral valve repair with rigid or semirigid ring? Ann Thorac Surg. 2014; 97(2): 492-7.

[4] Chung JW, Shin JK, Song MG, Chee HK, Kim JS. Patch valvuloplasty in patients with posterior chordae rupture. Int J Cardiol. 2012; 154(2): 206-7.

[5] Kim KH, Kim MH, Kim WH. Improvement of mitral valve coaptation with supraannular plication of the posterior annulus: A newly designed strip for posterior annular plication. Ann Thorac Cardiovasc Surg. 2012; 18(2): 95-100.

[6] Calafiore AM, Di Mauro M, Iacò AL, Varone E, Romeo A, Mangiafico S, et al. Resecting and nonresecting techniques for posterior mitral leaflet prolapse. J Card Surg. 2011; 26(2): 119-23.

[7] Maisano F, Ziskind Z, Grimaldi A, Blasio A, Caldarola A, De Bonis M, La Canna G, Alfieri O. Selective reduction of the septolateral dimensions in functional mitral regurgitation by modified-shape ring annuloplasty. J Thorac Cardiovasc Surg. 2005; 129(2): 472-4.

[8] De Bonis M, Taramasso M, Grimaldi A, Maisano F, Calabrese MC, Verzini A, et al. The GeoForm annuloplasty ring for the surgical treatment of functional mitral regurgitation in advanced dilated cardiomyopathy. Eur J Cardiothorac Surg. 2011; 40(2): 488-95.

[9] Robb JD, Minakawa M, Koomalsingh KJ, Shuto T, Jassar AS, Ratcliffe SJ, et al. Posterior leaflet augmentation improves leaflet tethering in repair of ischemic mitral regurgitation. Eur J Cardiothorac Surg. 2011; 40(6): 1501-7.

[10] Nouri M, Tokaldany ML, Shahrzad M, Mardanloo AS, Ahmadi SH, Roomi ZS, Sadeghian H. Echocardiographic determinants of ischemic mitral regurgitation. J Card Surg. 2013; 28(4): 359-65. 